\title{
Article \\ Admission Hydration Status and Ischemic Stroke Outcome-Experience from a National Registry of Hospitalized Stroke Patients
}

\author{
Yoav Eizenberg ${ }^{1}$, Ehud Grossman ${ }^{2}{ }^{\mathbb{D}}$, David Tanne ${ }^{3}$ and Silvia Koton ${ }^{4, *}$ \\ 1 Department of Endocrinology, Clalit Health Services, Sackler Faculty of Medicine, Tel Aviv University, Tel \\ Aviv-Yaffo District, Tel Aviv 6997801, Israel; yoave1@yahoo.com \\ 2 Internal Medicine Wing, The Chaim Sheba Medical Center, Sackler Faculty of Medicine, Tel Aviv University, \\ Tel Hashomer, Tel Aviv 6997801, Israel; ehud.grossman@sheba.health.gov.il \\ 3 Rambam Health Care Campus, Stroke and Cognition Institute, Haifa 3109601, Israel; \\ d_tanne@rambam.health.gov.il \\ 4 Stanley Steyer School of Health Professions, Sackler Faculty of Medicine, Tel-Aviv University, \\ Tel Aviv 6997801, Israel \\ * Correspondence: koton@tauex.tau.ac.il
}

Citation: Eizenberg, Y.; Grossman, E.; Tanne, D.; Koton, S. Admission

Hydration Status and Ischemic Stroke Outcome-Experience from a National Registry of Hospitalized Stroke Patients. J. Clin. Med. 2021, 10, 3292. https://doi.org/10.3390/ jcm10153292

Academic Editor: Georgios Tsivgoulis

Received: 18 April 2021

Accepted: 22 July 2021

Published: 26 July 2021

Publisher's Note: MDPI stays neutral with regard to jurisdictional claims in published maps and institutional affiliations.

Copyright: (c) 2021 by the authors. Licensee MDPI, Basel, Switzerland. This article is an open access article distributed under the terms and conditions of the Creative Commons Attribution (CC BY) license (https:// creativecommons.org/licenses/by/ $4.0 /)$.

\begin{abstract}
Reduced intravascular volume upon ischemic stroke (IS) admission has been associated with in-hospital complications, disability, and reduced survival. We aimed to evaluate the association of the urea-to-creatinine ratio (UCR) with disability or death at discharge, length of stay, in-hospital complications, and mortality during the first year. Using a national registry, we identified hospitalized IS patients without renal failure. Disability or death at discharge, length of stay, in-hospital complications, and mortality during the first year were studied by UCR, and associations between UCR levels and each outcome were assessed adjusting for age, sex, stroke severity, comorbidities, use of statins, and use of diuretics. In total, 2212 patients were included. Levels (median (25-75\%)) for the main study variables were: urea 5.16 (3.66-6.83) mmol/L; creatinine 80 (64-92) $\mu \mathrm{mol} / \mathrm{L}$; and UCR 65 (58-74). Levels of UCR were significantly higher in patients with disability or death at discharge $(p<0.0001)$, those with complications during hospitalization $(p=0.03)$, those with infection during hospitalization $(p=0.0003)$, and those dead at 1 year $(p<0.0001)$. Analysis by UCR quartile showed that rates of disability or death at discharge, infections, complications overall, and death at 1 year in patients with UCR in the 4th quartile were significantly higher than in others. Risk-factor-adjusted analysis by UCR quartiles demonstrated an inconsistent independent association between UCR and disability or death after ischemic stroke. A high 1-year mortality rate was observed in IS patients with elevated UCR, yet this finding was not statistically significant after controlling for risk factors. Our study shows inconsistent associations between hydration status and poor functional status at discharge, and no association with length of stay, in-hospital complications (infectious and overall), and 1-year mortality.
\end{abstract}

Keywords: stroke; dehydration; outcomes; complications; disability; death; creatinine

\section{Introduction}

Reduced intravascular volume, commonly referred to as dehydration, is a common finding upon admission of patients with ischemic stroke (IS), reported in 29-70\% of patients [1]. It has been associated with in-hospital complications [2-9], poor functional outcome at discharge [4,10,11], poor functional outcome at 30 days [12], and reduced survival at 90 days $[13,14]$. Current guidelines for treatment of IS recommend maintaining euvolemia [15], but accurately determining the intravascular status in IS patients remains a difficult task. Several methods to assess intravascular volume have been suggested; however, the blood urea nitrogen (BUN)/creatinine ratio and the urea/creatinine ratio (UCR) continue to be the most commonly used and widely accepted [1]. 
Using data from the National Acute Stroke Israeli Survey (NASIS) registry of hospitalized stroke patients, we aimed to evaluate the association of hydration status in IS, as measured by UCR, with stroke severity on admission, in-hospital complications, and mortality during the first year after ischemic stroke.

\section{Materials and Methods}

\subsection{Study Design and Setting}

The NASIS prospective hospital-based national registry was prospectively performed during 2-month periods triennially [16,17] (February-March 2004, March-April 2007, April-May 2010, April-May 2013, and April-May 2016). All consecutive patients hospitalized due to acute stroke or transient ischemic attack age 18 and over nationwide were included. The NASIS registry was approved by the ethical committees of the participating medical centers.

Data on creatinine and urea levels were collected in NASIS 2013 and 2016. In total, 4617 patients were included in these two periods, among them 3171 patients with ischemic stroke. Complete data on creatinine and urea levels were available for 2767/3171 (87.3\%). No significant differences between patients with and without data on urea and creatinine levels were observed in the distribution of demographics (age $(p=0.52)$, sex $(p=0.73)$ ), or in risk factors and clinical characteristics including hypertension $(p=0.99)$, diabetes $(p=0.77)$, current smoking $(p=0.89)$, prior stroke $(p=0.40)$, prior heart disease $(p=0.62)$, use of statins $(p=0.29)$, and use of diuretics $(p=0.19)$. We excluded 555 participants with renal failure (by history or with creatinine $>1.5 \mathrm{mg} / \mathrm{dL}$ ). Therefore, the final sample for the present analysis included 2212 ischemic stroke patients.

\subsection{Data Collection}

Coordinating physicians prospectively collected data from all the departments of the participating medical centers. A structured data form including demographics, risk factors and co-morbidities, stroke characteristics, in-hospital management, and outcome was used for collecting the information. Urea and creatinine levels were measured at local labs of all medical centers on admission. Mortality was prospectively assessed at 1 year after stroke by means of matching patients' files with national mortality data.

\subsection{Definition of Main Variables}

Urea/creatinine ratio (UCR) was used to evaluate level of hydration on admission. Severity of stroke on admission was categorized into five NIH Stroke Scale (NIHSS) [18] levels, as defined by the NASIS protocol. Severe stroke was defined as NIHSS $\geq 16$. Functional outcome was evaluated at discharge according to the modified Rankin Scale (mRS) [19] and categorized as mRS $0-1 ; 2-3 ; 4-5$; and 6 (death). Based on the mRS category, we defined disability at discharge or death as $\mathrm{mRS} \geq 2$ [20]. Complications during hospitalization were defined as neurological, cardiac, infectious, or bleeding during hospitalization. Infection included pneumonia, urinary tract infection, sepsis, fever, and other infection during hospitalization. Complications during hospitalization were reported at discharge and were analyzed overall and by studying infections as a separate outcome. We also assessed length of stay as a continuous variable and defined prolonged hospitalization as $\geq 7$ days.

\subsection{Statistical Analysis}

Baseline characteristics, stroke severity on admission, and complications during hospitalization were studied by UCR quartiles, as well as by looking at UCR level as a continuous variable. UCR shows a distribution skewed to the right with a mean of 38.6 and a median of 36.7. Therefore, associations between UCR level and all complications and infections during hospitalization were studied with the Wilcoxon test, while the Spearman correlation test was used to assess association between UCR level and length of stay.

In the multivariate analysis, logistic regression models were used to study associations between UCR levels as a continuous variable as well as UCR quartiles and each of 
the outcome variables, controlling for potential confounders. Odds ratio (OR) and 95\% confidence interval $(95 \% \mathrm{CI})$ associated with 1-SD UCR for each outcome were presented, adjusted for age and sex (Model 1) and further accounting for the following risk factors, selected a priori, based on clinical discretion and recently published evidence [21]: stroke severity, hypertension, diabetes mellitus, smoking, history of atrial fibrillation, prior stroke, prior heart disease, peripheral artery disease, disability before the stroke (mRS $\geq 2$ before the stroke), use of statins, and use of diuretics for 1 week or more before symptoms onset, in addition to age and sex (Model 2). Risk estimates by UCR quartiles using the lowest UCR quartile as reference group were computed with logistic regression models adjusting for risk factors as described (Model 1 and Model 2).

In a sensitivity analysis, potential associations between UCR and the outcome variables were evaluated in 236/2212 (10.7\%) patients undergoing reperfusion therapy (either intravenous thrombolysis or mechanical thrombectomy).

Analyses were performed with SAS 9.4 (SAS, SAS Institute, Cary, NC, USA).

\section{Results}

\subsection{Participants' Characteristics}

In total, 2212 ischemic stroke patients were included, with $47.6 \%$ being women. Mean (SD) age was 70.3 (13.2) years. Median levels (25-75\%) for the main study variables were as follows: urea $5.16(3.66-6.83) \mathrm{mmol} / \mathrm{L}(31(22-41) \mathrm{mg} / \mathrm{dL})$; creatinine 80 (64-92) $\mu \mathrm{mol} / \mathrm{L}$ (0.90 (0.72-1.04) mg/dL); UCR 65 (57-74) (36.69 (25.00-47.77)); and length of stay 5 (3-9) days. Severe stroke was reported for $8.4 \%$ of patients, while $65.6 \%$ were diagnosed with minor stroke (NIHSS $\leq 5$ ). Complications during hospitalization were observed in $403(18.2 \%)$ participants, while $242(11.0 \%)$ participants were diagnosed with infections and 260 (11.8\%) died during the first year after IS.

Characteristics of patients by UCR quartile are shown in Table 1. In general, rates of cardiovascular risk factors, except for smoking at time of stroke, were higher in participants in the highest UCR quartile compared with lower quartiles. Statistically significant differences in baseline characteristics by UCR quartiles were observed for demographics and most clinical factors, as shown in Table 1.

Table 1. Baseline characteristics of ischemic stroke patients by urea/creatinine ratio (UCR) quartiles.

\begin{tabular}{lccccc}
\hline & \multicolumn{4}{c}{ UCR Quartiles } & $p$ for Trend \\
\cline { 2 - 6 } & $\mathbf{n}=\mathbf{5 7 5 9}$ & $\mathbf{5 7 - 6 5}$ & $\mathbf{6 5 - 7 4}$ & $\mathbf{7 7 4}$ & \\
& $\mathbf{n}=\mathbf{5 4 7}$ & $\boldsymbol{n}=\mathbf{5 5 3}$ & $\boldsymbol{n}=\mathbf{5 5 3}$ & \\
\hline Age (SD), years & 68.10 & 67.43 & 71.14 & 74.33 & $<0.0001$ \\
Women & $(13.15)$ & $(13.23)$ & $(12.79)$ & $(12.48)$ & $<0.0001$ \\
Stroke severity by NIHSS & $229(41.0)$ & $213(38.9)$ & $245(44.3)$ & $366(66.2)$ & $<0.0001$ \\
$\leq 5$ & & & & & \\
$6-10$ & $399(71.4)$ & $373(68.2)$ & $363(65.6)$ & $315(57.0)$ & \\
$11-15$ & $100(17.9)$ & $98(17.9)$ & $116(21.0)$ & $120(21.7)$ & \\
$16-20$ & $25(4.5)$ & $37(6.8)$ & $39(7.1)$ & $41(7.4)$ & \\
20 & $22(3.9)$ & $24(4.4)$ & $20(3.6)$ & $55(10.0)$ & \\
Severe stroke (NIHSS $\geq 16)$ & $13(2.3)$ & $15(2.7)$ & $15(2.7)$ & $22(4.0)$ & \\
Hypertension & $35(6.3)$ & $39(7.1)$ & $35(6.3)$ & $77(13.9)$ & $<0.0001$ \\
Diabetes & $414(74.3)$ & $401(73.7)$ & $439(79.8)$ & $450(81.8)$ & 0.0003 \\
Current smoking & $244(43.7)$ & $218(39.9)$ & $231(41.9)$ & $245(44.5)$ & 0.66 \\
Prior stroke & $144(26.1)$ & $144(26.8)$ & $128(23.6)$ & $95(17.4)$ & 0.0003 \\
Atrial fibrillation & $114(20.6)$ & $150(27.6)$ & $155(28.2)$ & $153(28.5)$ & 0.0034 \\
Prior heart disease & $67(12.1)$ & $98(18.0)$ & $112(20.5)$ & $155(28.1)$ & $<0.0001$ \\
Peripheral arterial disease & $136(24.3)$ & $132(24.1)$ & $146(26.4)$ & $145(26.2)$ & 0.34 \\
Disability before the stroke & $20(3.6)$ & $23(4.2)$ & $24(4.4)$ & $39(7.1)$ & 0.009 \\
Statin medication use & $116(21.3)$ & $129(24.4)$ & $165(30.7)$ & $220(41.6)$ & $<0.0001$ \\
Diuretics use & $274(50.9)$ & $261(50.3)$ & $261(50.4)$ & $272(51.6)$ & 0.83 \\
\hline
\end{tabular}

Figures represent number and percentage if not otherwise specified. Prior heart disease was defined as chronic heart failure, prior myocardial infarction, atrial fibrillation, or valve disease. Disability before the stroke was defined as $\mathrm{mRS} \geq 2$ before stroke. Use of statin medication and diuretics was defined as treatment for at least 7 days before onset of stroke symptoms. 


\subsection{Associations Between UCR and Poor Outcome after Stroke}

Levels of UCR were significantly higher in patients with complications during hospitalization $(p=0.03)$, those with infection during hospitalization $(p=0.0003)$, those with disability or death at discharge $(p<0.0001)$, and those dead at 1 year $(p<0.0001)$. No correlation was observed between UCR and length of stay $(\mathrm{r}=0.01, p=0.56)$ or prolonged hospitalization ( $\geq 7$ days, $p=0.46$ ).

Analysis by UCR quartile showed that the rate of death at 1 year in patients with UCR in the 4th quartile was higher than in others (Table 2). Statistically significant differences in rates of outcome variables by UCR quartiles were observed for disability, infection during hospitalization, and 1-year mortality (Table 2).

Table 2. Functional status, length of stay, complications during hospitalization, and 1-year mortality in ischemic stroke patients by urea/creatinine ratio (UCR) level.

\begin{tabular}{|c|c|c|c|c|c|}
\hline & \multicolumn{4}{|c|}{ UCR QUARTILES } & \multirow{2}{*}{$\begin{array}{l}p \text { for } \\
\text { Trend }\end{array}$} \\
\hline & $\begin{array}{c}<57 \\
n=559\end{array}$ & $\begin{array}{c}57-65 \\
n=547\end{array}$ & $\begin{array}{c}65-74 \\
n=553\end{array}$ & $\begin{array}{c}>74 \\
n=553\end{array}$ & \\
\hline Modified Rankin Scale (mRS) & & & & & $<0.0001$ \\
\hline $0-1$ & $285(51.08)$ & $241(44.14)$ & $222(40.14)$ & $182(32.91)$ & \\
\hline $2-3$ & $168(30.11)$ & $191(34.98)$ & $201(36.35)$ & $163(29.48)$ & \\
\hline $4-5$ & $85(15.23)$ & $95(17.40)$ & $115(20.80)$ & $176(31.83)$ & \\
\hline 6 & $20(3.58)$ & $19(3.48)$ & $15(2.71)$ & $32(5.79)$ & \\
\hline Disability or death (discharge $m R S \geq 2$ ) & $273(48.92)$ & $305(55.86)$ & $331(59.86)$ & $371(67.90)$ & $<0.0001$ \\
\hline Length of stay, mean (SD), days & $8.42(18.74)$ & $7.80(8.51)$ & $7.47(9.10)$ & $9.24(18.52)$ & 0.19 \\
\hline Complications during hospitalization & $99(17.74)$ & $92(16.85)$ & $86(15.55)$ & $126(22.83)$ & 0.06 \\
\hline Infection during hospitalization & $53(9.50)$ & $48(8.79)$ & $55(9.95)$ & $86(15.61)$ & 0.0011 \\
\hline 1-year mortality & $61(12.45)$ & 50 (10.99) & $58(13.52)$ & $91(20.97)$ & 0.0002 \\
\hline
\end{tabular}

Figures represent number and percentage if not otherwise specified.

Our findings suggest non-linear associations between UCR levels and length of stay and complications during hospitalization (Table 2).

Results of the multivariate analysis of associations between level of UCR and the studied outcomes show that following adjustment for age, sex, stroke severity, and cardiovascular risk factors (Model 2), no significant associations were observed between UCR as a continuous variable and stroke outcome (Table 3).

Table 3. Stroke outcome estimates (ORs $(95 \%$ Cis)) associated with level of urea/creatinine ratio (UCR).

\begin{tabular}{lccc}
\hline & \multicolumn{3}{c}{ OR (95\% CI) for 1-SD in UCR } \\
\hline & Unadjusted & Model 1 & Model 2 \\
\hline Disability or death (discharge mRS $\geq 2)$ & $\mathbf{1 . 3 8 ( 1 . 2 3 - 1 . 5 5 )}$ & $\mathbf{1 . 1 8 ( 1 . 0 6 - 1 . 3 1 )}$ & $1.07(0.97-1.19)$ \\
Length of stay $\geq 7$ days & $1.01(0.93-1.10)$ & $0.98(0.90-1.07)$ & $0.95(0.85-1.05)$ \\
Complications during hospitalization & $1.07(0.97-1.18)$ & $0.99(0.88-1.11)$ & $0.85(0.72-1.01)$ \\
Infection during hospitalization & $\mathbf{1 . 1 4}(\mathbf{1 . 0 3 - 1 . 2 7 )}$ & $1.07(0.95-1.21)$ & $0.95(0.78-1.17)$ \\
1-year mortality & $\mathbf{1 . 1 9}(\mathbf{1 . 0 6}-\mathbf{1 . 3 4})$ & $1.11(0.97-1.27)$ & $0.99(0.81-1.21)$ \\
\hline
\end{tabular}

Model 1 adjusted for age and sex. Model 2 adjusted for age, sex, stroke severity, hypertension, diabetes, current smoking, atrial fibrillation, prior stroke, prior heart disease, peripheral artery disease, disability before the stroke, use of statins, and use of diuretics for1 week or more before symptoms onset. Statistically significant estimates are shown in bold.

In the analysis by UCR quartiles, age- and sex-adjusted estimates (ORs, 95\% CIs) for disability or death at discharge were increased for patients with UCR in the 2nd, $3 \mathrm{rd}$, and 4th quartiles, compared with those in the first quartile. These findings were consistent after controlling for risk factors (Model 2) for patients in the 2nd quartile of UCR (Table 4). Unadjusted estimates (ORs, 95\% CIs) for in-hospital complications, infections, 
and 1-year mortality were increased for patients in the 4th compared with the 1st UCR quartile (Table 4). However, these findings were not consistent following adjustment (Table 4, Model 2). Interestingly, a negative association between complications and UCR was observed for patients in the 3rd quartile compared with the 1st quartile (adjusted OR $0.66,95 \%$ CI 0.45-0.97) (Table 4).

Table 4. Stroke outcome estimates (ORs (95\% Cis)), by quartiles of urea/creatinine ratio (UCR).

\begin{tabular}{|c|c|c|c|c|}
\hline & \multicolumn{4}{|c|}{ OR $(95 \% \mathrm{CI})$} \\
\hline & UCR & Unadjusted & Model 1 & Model 2 \\
\hline \multirow{4}{*}{ Disability or death (discharge $\mathrm{mRS} \geq 2$ ) } & 1st quartile & 1 (Reference) & 1 (Reference) & 1 (Reference) \\
\hline & 2nd quartile & $1.32(1.04-1.67)$ & $1.44(1.12-1.85)$ & $1.38(1.01-1.91)$ \\
\hline & 3rd quartile & $1.56(1.23-1.97)$ & $1.38(1.07-1.77)$ & $1.21(0.87-1.67)$ \\
\hline & 4th quartile & $2.13(1.67-2.71)$ & $1.59(1.22-2.06)$ & $1.14(0.80-1.63)$ \\
\hline \multirow{4}{*}{ Length of stay $\geq 7$ days } & 1st quartile & 1 (Reference) & 1 (Reference) & 1 (Reference) \\
\hline & 2nd quartile & $0.94(0.69-1.28)$ & $0.99(0.78-1.26)$ & $0.92(0.70-1.20)$ \\
\hline & 3rd quartile & $0.85(0.62-1.17)$ & $0.83(0.65-1.06)$ & $0.62(0.58-1.02)$ \\
\hline & 4th quartile & $1.37(1.02-1.84)$ & $0.94(0.74-1.21)$ & $0.77(0.58-1.02)$ \\
\hline \multirow{4}{*}{ Complications during hospitalization } & 1st quartile & 1 (Reference) & 1 (Reference) & 1 (Reference) \\
\hline & 2nd quartile & $0.94(0.69-1.28)$ & $0.97(0.70-1.33)$ & $0.82(0.56-1.19)$ \\
\hline & 3rd quartile & $0.84(0.61-1.15)$ & $0.75(0.54-1.03)$ & $0.66(0.45-0.97)$ \\
\hline & 4th quartile & $1.37(1.02-1.84)$ & $1.04(0.76-1.41)$ & $0.76(0.53-1.11)$ \\
\hline \multirow{4}{*}{ Infection during hospitalization } & 1st quartile & 1 (Reference) & 1 (Reference) & 1 (Reference) \\
\hline & 2nd quartile & $0.92(0.61-1.38)$ & $0.95(0.62-1.44)$ & $0.74(0.44-1.23)$ \\
\hline & 3rd quartile & $1.05(0.71-1.57)$ & $0.91(0.60-1.39)$ & $0.78(0.47-1.28$ \\
\hline & 4th quartile & $1.76(1.22-2.54)$ & $1.26(0.86-1.85)$ & $0.91(0.57-1.45)$ \\
\hline \multirow{4}{*}{ 1-year mortality } & 1st quartile & 1 (Reference) & 1 (Reference) & 1 (Reference) \\
\hline & 2nd quartile & $0.87(0.58-1.29)$ & $0.89(0.59-1.35)$ & $0.72(0.43-1.21)$ \\
\hline & 3rd quartile & $1.10(0.75-1.62)$ & $0.88(0.59-1.32)$ & $0.83(0.51-1.35)$ \\
\hline & 4th quartile & $1.87(1.31-2.66)$ & $1.29(0.88-1.88)$ & $0.97(0.61-1.55)$ \\
\hline
\end{tabular}

Model 1 adjusted for age and sex. Model 2 adjusted for age, sex, stroke severity, hypertension, diabetes, current smoking, atrial fibrillation, prior stroke, prior heart disease, peripheral artery disease, disability before the stroke, use of statins, and use of diuretics for1 week or more before symptoms onset. Statistically significant estimates are shown in bold.

In the sensitivity analysis of data on patients undergoing reperfusion therapy during hospitalization, no significant associations were observed between UCR level and the study outcomes in the unadjusted models or in models adjusted for age and sex (Model 1). We present the results of this analysis in Table 5. Due to the small number of patients with reperfusion therapy, further adjustment was not conducted in the regression models.

Table 5. Stroke outcome estimates (ORs (95\% Cis)) associated with level of urea/creatinine ratio (UCR) in patients treated with reperfusion therapy, $n=236$.

\begin{tabular}{lcc}
\hline & \multicolumn{1}{c}{ OR (95\% CI) for 1-SD in UCR } \\
\hline & Unadjusted & Model 1 \\
\hline Disability or death (discharge mRS $\geq 2)$ & $0.85(0.59-1.23)$ & $0.74(0.50-1.11)$ \\
Length of stay $\geq 7$ days & $0.99(0.69-1.42)$ & $0.90(0.62-1.32)$ \\
Complications during hospitalization & $1.24(0.85-1.82)$ & $1.14(0.76-1.71)$ \\
Infection during hospitalization & $1.39(0.91-2.14)$ & $1.34(0.85-2.09)$ \\
1-year mortality & $0.97(0.56-1.71)$ & $0.84(0.45-1.54)$ \\
\hline
\end{tabular}

Model 1 adjusted for age and sex. 


\section{Discussion}

Dehydration is common in IS patients and has been associated with several unfavorable outcomes. Yet, our study shows inconsistent association between hydration status and poor functional status at discharge and no association with length of stay, in-hospital complications (infectious and overall), and 1-year mortality.

Dehydration, characterized by an elevated UCR, has been linked to disability or death at discharge in several studies to this point. Compatible with our study, Rowat et al. showed that a UCR $>80(\mathrm{UCR}>45)$ was associated with dependency or death at hospital discharge [10]. However, in our study, the risk for a poor functional outcome was significant at a lower UCR, with loss of significance in higher UCR quartiles. In another study, IS patients with a BUN/creatinine ratio $>26$ (BUN/Creatinine ratio $>15$ ) (equivalent to a UCR $>56$ $(\mathrm{UCR} \geq 32)$ ) had lower mRS scores even after controlling for age, sex, hypertension, diabetes, admission NIHSS score, and infection, but no other UCR cutoffs were explored [4]. It is noteworthy that the sensitivity analysis including only patients with reperfusion therapy did not show associations between UCR and any of the study outcomes, as was also demonstrated by $\mathrm{Wu}$ et al. with a larger sample [11].

Our results do not support the association of UCR with length of stay. Smithard et al. investigated the relationship of dysphagia, a common pathophysiological cause of dehydration in IS, with length of stay and also did not found an association [22]. A more recent interventional study showed a shorter length of stay in IS patients given intravenous saline based on a BUN/creatinine ratio $>26(\mathrm{BUN} /$ creatinine ratio $>15)$ (equivalent to a $\mathrm{UCR}>56(\mathrm{UCR} \geq 32)$ ) as a marker of dehydration [23]. This study also reported a reduced infection rate in patients receiving intravenous saline based on the same BUN/creatinine ratio cutoff. Moreover, in a large cohort study by Liu et al., a higher in-hospital infection rate was observed in IS patients with an elevated BUN/creatinine ratio [4]. However, our data reflect a stable infection rate over a wide range of UCRs. This may be due to differences in studies' populations or in local acute care procedures. Our data also show that overall in-hospital complications are not associated with hydration status.

After adjusting for several potential confounders, we found that dehydration was not associated with increased mortality in the first year after the stroke admission. On the contrary, Bhalla et al. found that increased plasma osmolality is significantly associated with mortality at 90 days, although with borderline significance [13], possibly attributed to a small number of patients. Moreover, data adapted from a large prospective trial showed that mortality at 90 days was associated with an elevated urea level but not with an elevated UCR [14], perhaps due to concomitant underlying renal dysfunction [24]. To the best of our knowledge, previous studies have not recorded mortality data for longer periods.

Our study has several strengths. First, it uses a national registry of considerable size including all hospitalized stroke patients in the relevant time periods. Second, hospitalization data were collected by well-trained experienced teams, minimizing data mishandling. Third, stroke severity on admission and functional status were assessed using validated quantitative measures (NIHSS and mRS, respectively). However, the present study has some limitations. NASIS is a hospital-based registry; therefore, it is possible that minor ischemic strokes not referred to the hospital were not recorded, although hospitalization of all stroke cases is the current policy in Israel. Hydration status was pragmatically defined by UCR, not by more reliable measures such as plasma osmolarity. In addition, data on pre-admission factors (e.g., time to admission) and post-admission factors (e.g., varying hydration protocols among participating hospitals) that may affect hydration status were only partially available in NASIS. Although NASIS includes broad patient-level information, data on NIHSS score as a continuous variable as well as severity or duration of baseline medical conditions were not available. Lastly, residual confounding is a possibility as in any observational study. 


\section{Conclusions}

Our study shows inconsistent associations between hydration status and poor functional status at discharge and no association with length of stay, in-hospital complications (infectious and overall), and 1-year mortality. As current literature continues to be inconclusive on this matter, large prospective studies are needed to reach more firm conclusions.

Author Contributions: Formal analysis, S.K.; writing—original draft preparation, Y.E., S.K.; writingreview and editing, E.G., D.T. All authors have read and agreed to the published version of the manuscript.

Funding: This research received no external funding.

Institutional Review Board Statement: The study was conducted according to the guidelines of the Declaration of Helsinki, and approved by the Institutional Review Board (or Ethics Committee) of all participating hospitals.

Informed Consent Statement: Patient consent was waived by the relevant Institutional Review Boards due to the NASIS anonymous and retrospective properties.

Data Availability Statement: The data presented in this study are available on request from the corresponding author. The data are not publicly available due to privacy restrictions.

Conflicts of Interest: The authors declare no conflict of interest.

\section{References}

1. Bahouth, M.N.; Gottesman, R.F.; Szanton, S.L. Primary 'dehydration' and acute stroke: A systematic research review. J. Neurol. 2018, 265, 2167-2181. [CrossRef]

2. Lin, L.C.; Yang, J.; Weng, H.-H.; Hsiao, C.; Lai, S.L.; Fann, W. Predictors of early clinical deterioration after acute ischemic stroke. Am. J. Emerg. Med. 2011, 29, 577-581. [CrossRef] [PubMed]

3. Lin, L.; Fann, W.; Chou, M.; Chen, H.; Su, Y.; Chen, J. Urine specific gravity as a predictor of early neurological deterioration in acute ischemic stroke. Med. Hypotheses 2011, 77, 11-14. [CrossRef]

4. Liu, C.-H.; Lin, S.-C.; Lin, J.-R.; Yang, J.-T.; Chang, Y.-J.; Chang, C.-H.; Chang, T.-Y.; Huang, K.-L.; Ryu, S.-J.; Lee, T.-H. Dehydration is an independent predictor of discharge outcome and admission cost in acute ischaemic stroke. Eur. J. Neurol. 2014, 21, $1184-1191$. [CrossRef] [PubMed]

5. Mohanty, S.; Tripathi, B.K.; Bhatia, K.; Gupta, B.; Mittal, M.K. Predictors of early neurological deterioration in patients with acute ischaemic stroke with special reference to blood urea nitrogen (BUN)/creatinine ratio \& urine specific gravity. Indian J. Med. Res. 2015, 141, 299-307. [CrossRef]

6. Bahouth, M.N.; Bahrainwala, Z.; Hillis, A.E.; Gottesman, R.F. Dehydration Status is Associated with More Severe Hemispatial Neglect After Stroke. Neurologist 2016, 21, 101-105. [CrossRef]

7. Li, S.-S.; Yin, M.-M.; Zhou, Z.-H.; Chen, H.-S. Dehydration is a strong predictor of long-term prognosis of thrombolysed patients with acute ischemic stroke. Brain Behav. 2017, 7, e00849. [CrossRef] [PubMed]

8. Deng, L.; Qiu, S.; Wang, C.; Bian, H.; Wang, L.; Li, Y.; Wu, B.; Liu, M. Effects of the blood urea nitrogen to creatinine ratio on haemorrhagic transformation in AIS patients with diabetes mellitus. BMC Neurol. 2019, 19, 63. [CrossRef]

9. Shi, Z.; Zheng, W.C.; Yang, H.; Fu, X.L.; Cheng, W.Y.; Yuan, W.J. Contribution of dehydration to END in acute ischemic stroke not mediated via coagulation activation. Brain Behav. 2019, 9, e01301. [CrossRef]

10. Rowat, A.; Graham, C.; Dennis, M. Dehydration in Hospital-Admitted Stroke Patients. Stroke 2012, 43, 857-859. [CrossRef] [PubMed]

11. Wu, F.-F.; Hung, Y.-C.; Tsai, Y.H.; Yang, J.-T.; Lee, T.-H.; Liow, C.-W.; Lee, J.-D.; Lin, C.-J.; Peng, T.-I.; Lin, L.-C. The influence of dehydration on the prognosis of acute ischemic stroke for patients treated with tissue plasminogen activator. BMC Cardiovasc. Disord. 2017, 17, 154. [CrossRef]

12. Schrock, J.W.; Glasenapp, M.; Drogell, K. Elevated blood urea nitrogen/creatinine ratio is associated with poor outcome in patients with ischemic stroke. Clin. Neurol. Neurosurg. 2012, 114, 881-884. [CrossRef] [PubMed]

13. Bhalla, A.; Sankaralingam, S.; Dundas, R.; Swaminathan, R.; Wolfe, C.D.A.; Rudd, A.G. Influence of raised plasma osmolality on clinical outcome after acute stroke. Stroke 2000, 31, 2043-2048. [CrossRef] [PubMed]

14. Billington, C.K.; Appleton, J.P.; Berge, E.; Sprigg, N.; Glover, M.; Bath, P.M.W. Impact of hydration status on haemodynamics, effects of acute blood pressure-lowering treatment, and prognosis after stroke. Br. J. Clin. Pharmacol. 2018, 84, $2914-2922$. [CrossRef]

15. Powers, W.J.; Rabinstein, A.A.; Ackerson, T.; Adeoye, O.M.; Bambakidis, N.C.; Becker, K.; Biller, J.; Brown, M.; Demaerschalk, B.M.; Hoh, B.; et al. 2018 Guidelines for the Early Management of Patients with Acute Ischemic Stroke: A Guideline for Healthcare Professionals From the American Heart Association/American Stroke Association. Stroke 2018, 50, e277-e278. [CrossRef] 
16. Tanne, D.; Goldbourt, U.; Koton, S.; Grossman, E.; Koren-Morag, N.; Green, M.S.; Bornstein, N.M. National Acute Stroke Israeli Survey Group a National Survey of Acute Cerebrovascular Disease in Israel: Burden, Management, Outcome and Adherence to Guidelines. Isr. Med. Assoc. J. 2006, 8, 3-7.

17. Koton, S.; Tanne, D.; Green, M.S.; Bornstein, N.M. Mortality and Predictors of Death 1 Month and 3 Years after First-Ever Ischemic Stroke: Data from the First National Acute Stroke Israeli Survey (NASIS 2004). Neuroepidemiology 2010, 34, 90-96. [CrossRef]

18. National Institute of Neurological Disorders and Stroke rt-PA Stroke Study Group. Tissue Plasminogen Activator for Acute Ischemic Stroke. N. Engl. J. Med. 1995, 333, 1581-1588. [CrossRef] [PubMed]

19. Herndon, R.M. (Ed.) Handbook of Neurologic Rating Scales; Demos Vermande: New York, NY, USA, 1997; ISBN 978-1-888799-07-1.

20. Koton, S.; Telman, G.; Kimiagar, I.; Tanne, D. Gender differences in characteristics, management and outcome at discharge and three months after stroke in a national acute stroke registry. Int. J. Cardiol. 2013, 168, 4081-4084. [CrossRef] [PubMed]

21. Torres-Aguila, N.P.; Carrera, C.; Muiño, E.; Cullell, N.; Carcel-Marquez, J.; Gallego-Fabrega, C.; González-Sánchez, J.; Bustamante, A.; Delgado, P.; Ibañez, L.; et al. Clinical Variables and Genetic Risk Factors Associated with the Acute Outcome of Ischemic Stroke: A Systematic Review. J. Stroke 2019, 21, 276-289. [CrossRef]

22. Smithard, D.; O’Neill, P.; Park, C.; Morris, J.; Wyatt, R.; England, R.; Martin, D. Complications and Outcome After Acute Stroke. Stroke 1996, 27, 1200-1204. [CrossRef] [PubMed]

23. Lin, W.-C.; Shih, H.-M.; Lin, L.-C. Preliminary Prospective Study to Assess the Effect of Early Blood Urea Nitrogen/Creatinine Ratio-Based Hydration Therapy on Poststroke Infection Rate and Length of Stay in Acute Ischemic Stroke. J. Stroke Cerebrovasc. Dis. 2015, 24, 2720-2727. [CrossRef] [PubMed]

24. Kim, H.J.; Kim, J.-K.; Oh, M.S.; Kim, S.G.; Yu, K.-H.; Lee, B.-C. A Low Baseline Glomerular Filtration Rate Predicts Poor Clinical Outcome at 3 Months after Acute Ischemic Stroke. J. Clin. Neurol. 2015, 11, 73-79. [CrossRef] [PubMed] 